Vietnam Journal of Mechanics, VAST, Vol. 42, No. 1 (2020), pp. 1 -14

DOI: https://doi.org/10.15625/0866-7136/14699

\title{
A COMPREHENSIVE REVIEW ON DUAL APPROACH TO THE VIBRATION ANALYSIS: SOME DUAL TECHNIQUES AND APPLICATION
}

\author{
N. D. $\operatorname{Anh}^{1,2}$ \\ ${ }^{1}$ Institute of Mechanics, VAST, Hanoi, Vietnam \\ ${ }^{2}$ VNU University of Engineering and Technology, Hanoi, Vietnam \\ E-mail: ndanh@imech.vast.vn
}

Received: 16 October 2019 / Published online: 13 December 2019

\begin{abstract}
This paper reviews key ideas of the researches on the dual approach to the vibration analysis. Three types of dual techniques, namely, forward - return dual technique, global-local dual technique, weighted averaging dual technique, for the problem of equivalent replacement are summarized. Different implements and realizations of dual techniques to nonlinear vibration analysis and design of dynamic absorbers are reviewed. Finally, the challenging issues based on the dual techniques are discussed. A number of possibilities for developing analytical techniques related to dual techniques are proposed.

Keywords: dual approach, dual technique, forward - return dual, global-local dual, weighted averaging dual.
\end{abstract}

\section{INTRODUCTION}

Oscillation plays an important role in our daily lives and nature. This reflects the fact that life and nature are extremely diverse sets of motions. Fidlin wrote "Any motion is deeply connected with one of the most fundamental properties of nature - its ability to react with oscillations at any internal change or external influence" [1]. In all areas of human activities vibrational systems are increasingly abundant and varied and the study of those systems is always required. Most phenomena in our world are essentially nonlinear and described by nonlinear equations. We might simplify nonlinear phenomena as linear ones to make them easier to understand; however, for further investigation nonlinear phenomena should be treated as nonlinear problems. Thus, the study of nonlinear problems is of crucial importance not only in all areas of physics but also in engineering and in other disciplines. In particular, it appears that the analysis of vibration based on nonlinear mathematical models requires appropriate methods. Therefore, new methods for analysis of nonlinear oscillations always cause concern of scientists and technicians. Recently, a dual approach has been proposed to study the response of nonlinear systems [2]

(c) 2020 Vietnam Academy of Science and Technology 
and some dual techniques have been developed, e.g. [3-27], based on the concept of duality. Nature and life always contain dual inclinations. Those are perspectives that are contradictory, or complementary to each other. Natural phenomena and human activities exhibit often dual characters which reflect two side processes or/and the relative balance of two opposite sides. For illustration we may say attack - defense in a football match, one way and return in an excursion, day and night. When a problem is considered it is quite often that one its side is given too much attention while another side is almost or completely forgotten. This usual approach doesn't reflect the real essence of the problem in question and hence doesn't yield an expected solution in many cases. The main issue of a dual approach to a scientific problem is to always consider two different (dual) aspects of the problem. This allows the study to become more harmonious and reflects the essence of the problem. In the dual approach a dual technique is one that can introduce two dual perspectives for the same problem in consideration. Let one needs to investigate a problem. The use of the dual approach to this problem means that one should introduce a dual technique that can reflect the essence of the problem.

This paper reviews key ideas of the reported researches on the dual approaches to the vibration analysis [3-27]. Various types of dual techniques, different implements and realizations of dual approaches to vibration analysis and control are reviewed. Finally, the challenging issues on the dual techniques are discussed.

\section{DUAL EQUIVALENT REPLACEMENT}

The first introduction of the dual approach to the nonlinear vibrations was suggested in [2] for the problem of equivalent linearization. In science we usually needs to replace approximately an object $A$ described by the function $A(x)$ with the object $B$ described by the function $B(x)$. Thus, we will replace $A(x)$ approximately with $k B(x)$ where $k$ is an equivalent replacement coefficient to be found from an equivalent replacement criterion, for example, by the mean-square error criterion

$$
\left\langle e^{2}\right\rangle=\left\langle(A(x)-k B(x))^{2}\right\rangle \rightarrow \min _{k}
$$

where $e=A(x)-k B(x)$ is the error of replacement, $\langle\cdot\rangle$ is the deterministic averaging operator or expectation operator for the case of random functions $A(x), B(x)$. If $B(x)$ is a linear function of $x, B(x)=x$, the equivalent replacement is called equivalent linearization and $k$ is called equivalent linearization coefficient.

\subsection{Forward - return dual technique}

It was observed in [2] that the conventional equivalent replacement has the one-way sense. Indeed the conventional replace of $A(x)$ by $k B(x)$ can be considered as the forward replacement from the original function $A(x)$ to its counterpart $B(x)$ and the equivalent replacement coefficient $k$ is found from criterion (1) which is rewritten as follows

$$
\left\langle e_{f}^{2}\right\rangle \equiv\left\langle(A(x)-k B(x))^{2}\right\rangle \rightarrow \min _{k} .
$$

Hereinafter the indexes " $f$ ", " $r$ " are introduced to denote the forward and return replacements, respectively. The criterion (2) gives the forward equivalent replacement 
coefficient

$$
k_{f}=\frac{\langle A(x) B(x)\rangle}{\left\langle B^{2}(x)\right\rangle} .
$$

In order to propose a dual technique to the problem of equivalent replacement one should look for another replacement which is complementary to the forward replacement. The first leg and second leg in a Champions League tie may be a good suggestion and the adding up the scores and away goals rule are interesting illustrations of how two replacements can be combined. One of natural solutions was suggested in [2] as the return replacement. Thus supposing now $k_{f} B(x)$ is found one gets back to $A(x)$ using the return replacement

$$
k_{f} B(x) \rightarrow \lambda A(x),
$$

where the return coefficient $\lambda$ is determined from the mean-square error criterion

$$
\left\langle e_{r}^{2}\right\rangle \equiv\left\langle(k B(x)-\lambda A(x))^{2}\right\rangle \rightarrow \min _{\lambda}
$$

where $k=k_{f}$. Using (5), (3) and (2), one obtains

$$
\lambda_{r}=k_{f} \frac{\langle A(x) B(x)\rangle}{\left\langle A^{2}(x)\right\rangle}=r^{2},
$$

where it is denoted

$$
r=\frac{\langle A(x) B(x)\rangle}{\sqrt{\left\langle A^{2}(x)\right\rangle\left\langle B^{2}(x)\right\rangle}} .
$$

The parameter $r$ is precisely the correlation coefficient that is used as a measure of the linear dependence between two functions $A(x), B(x)$ [28]. The minimal mean-square errors of forward and return replacements can be calculated, respectively,

$$
\begin{gathered}
\left\langle e_{f}^{2}\right\rangle_{\min }=\left\langle\left(A(x)-k_{f} B(x)\right)^{2}\right\rangle=\left\langle A^{2}(x)\right\rangle-2 k_{f}\langle A(x) B(x)\rangle+k_{f}^{2}\left\langle B^{2}(x)\right\rangle=\left(1-r^{2}\right)\left\langle A^{2}(x)\right\rangle, \\
\left\langle e_{r}^{2}\right\rangle_{\min }=\left\langle\left(k_{f} B(x)-\lambda_{r} A(x)\right)^{2}\right\rangle=k_{f}^{2}\left\langle B^{2}(x)\right\rangle-2 k_{f} \lambda_{r}\langle A(x) B(x)\rangle+\lambda_{r}^{2}\left\langle A^{2}(x)\right\rangle=r^{2}\left(1-r^{2}\right)\left\langle A^{2}(x)\right\rangle .
\end{gathered}
$$

Application of the dual approach to the problem of replacement proposes this problem should combine two forward and return replacements (1) and (5) in a weighted dual replacement characterized by a weighted dual mean-square error criterion $[2,10,23]$

$$
\left\langle e_{w}^{2}\right\rangle \equiv(1-p)\left\langle e_{f}^{2}\right\rangle+p\left\langle e_{r}^{2}\right\rangle=(1-p)\left\langle(A(x)-k B(x))^{2}\right\rangle+p\left\langle(k B(x)-\lambda A(x))^{2}\right\rangle \rightarrow \min _{k, \lambda}
$$

where the index " $w$ " denotes the weighted dual replacement, $p$ is a non-dimensional weight parameter, $0 \leq p \leq 1$, which adjusts the contributions of forward and return replacements in order to obtain the best replacement of $A(x)$ by $k B(x)$. The minimum necessary and sufficient conditions of (10) give $k_{w}$ and $\lambda_{w}$, respectively [23]

$$
k_{w}=\left(1-p+p \lambda_{w}\right) \frac{\langle A(x) B(x)\rangle}{\left\langle B^{2}(x)\right\rangle}, \quad \lambda_{w}=k_{w} \frac{\langle A(x) B(x)\rangle}{\left\langle A^{2}(x)\right\rangle} .
$$


It is seen from (11) that the return replacement contributes to the equivalent replacement coefficient $k_{w}$ through the return coefficient $\lambda_{w}$ that plays a complementary role. From (11) one gets

$$
k_{w}=\frac{1-p}{1-p r^{2}} \frac{\langle A(x) B(x)\rangle}{\left\langle B^{2}(x)\right\rangle}, \quad \lambda_{w}=\frac{1-p}{1-p r^{2}} r^{2} .
$$

The problem is now reduced to how the weight parameter $p$ can be chosen. It is observed in [23] that the return replacement $e_{r}$ is introduced in (10) due to that the minimal mean-square forward error $e_{f \text { min }}$ differs from zero. Hence, it is assumed that the weight parameter $p$ can be a linear function of $e_{f \min }$ as follows

$$
p=\eta e_{f \min }
$$

where $\eta$ is a dimensional coefficient which can be taken as an effect sum of square mean of the original function $A(x)$ and its counterpart $k_{f} B(x)$

$$
\eta=\frac{1}{\left\langle A^{2}\right\rangle+\left\langle\left(k_{f} B\right)^{2}\right\rangle}=\frac{1}{\left\langle A^{2}\right\rangle+\left(\frac{\langle A B\rangle}{\left\langle B^{2}\right\rangle}\right)^{2}\left\langle B^{2}\right\rangle}=\frac{1}{\left(1+r^{2}\right)\left\langle A^{2}\right\rangle} .
$$

Substituting (14), (8) into (13) yields the following expression for the non-dimensional weight parameter $p$ [23]

$$
p=\frac{1-r^{2}}{1+r^{2}}
$$

Substituting (15) into (12) yields the following weighted dual equivalent replacement coefficient

$$
k_{w}=\frac{2 r^{2}}{1+r^{4}} \frac{\langle A B\rangle}{\left\langle B^{2}\right\rangle} .
$$

Thus, using the weighted dual mean-square error criterion (10) with the weight parameter (15) the original random function $A(x)$ can be replaced by the equivalent random function $k_{w} B(x)$ where the weighted dual equivalent replacement coefficient $k_{w}$ is found from (16) and $r$ is determined by (7). When $p=0$ the criterion (10) leads to the conventional mean-square error criterion [29]; and when $p=1 / 2$ it leads to the dual mean-square error criterion investigated in $[4,5]$.

\subsection{Global-local dual technique}

In this subsection another dual technique is reviewed to show how the dual approach could be applied to the problem of equivalent replacement. In 1995, based on the assumption that the global integration domain taken in the mean square error criterion should be reduced to a local one where the response would be concentrated Anh and Di Paola [30] proposed a local mean square error criterion (LOMSEC). Further investigations [31,32] have shown an improved accuracy of this criterion; however, the local domain in question was unknown and it has resulted in the main disadvantage of LOMSEC. Using the dual approach to LOMSEC, one may suggest a consideration with respect to two aspects, namely to local and global levels. According to this concept new values of replacement coefficients were obtained as global averaged values of all local replacement 
coefficients. The technique obtaining new replacement coefficients is called global-local dual technique that is reviewed in details as follows. Let we replace $A(x)$ approximately with $k B(x)$ where $k$ is an equivalent replacement coefficient to be found from the meansquare error criterion (1). Suppose that $A(x)$ and $B(x)$ are random functions. Rewriting (1) in explicit form gives

$$
\int_{-\infty}^{+\infty}(A(x)-k B(x))^{2} P(x) \mathrm{d} x \rightarrow \min _{k}
$$

where $P(x)$ is the probabilistic density function (PDF) of $x$. Since the integrations are taken over the entire coordinate space $(-\infty+\infty)$, criterion (1) may be called as a global mean square error criterion. The question is how two dual aspects can be suggested to (17). For that purpose one replaces the global value $+\infty$ in (17) by a local positive value $x_{0}$ and that leads to the following local mean square error criterion (LOMSEC)

$$
\int_{-x_{0}}^{x_{0}}(A(x)-k B(x))^{2} P(x) \mathrm{d} x \rightarrow \min _{k} .
$$

The expected integrations in (18) can be transformed to non-dimensional variables by $x_{0}=r \sigma_{x}$ where $r$ is a positive value, $\sigma_{x}$ is the normal deviation of the random variable $x$. Thus, criterion (18) leads to

$$
\left[(A(x)-k B(x))^{2}\right] \equiv \int_{-r \sigma_{x}}^{+r \sigma_{x}}(A(x)-k B(x))^{2} P(x) \mathrm{d} x \rightarrow \min _{k},
$$

where [.] denotes the local mean value of random variable which is introduced $[9,14]$ as follows

$$
[.]=\int_{-r \sigma_{x}}^{+r \sigma_{x}}(.) P(x) \mathrm{d} x .
$$

Criterion (19) gives the following replacement coefficient similar to the case of the classical mean square error criterion

$$
k(r)=\frac{[A(x) B(x)]}{\left[B^{2}(x)\right]} .
$$

It is seen from (21) that the replacement coefficient obtained by LOMSEC is function depending on $r$. In this sense, the replacement coefficient $k(r)$ is called as local replacement coefficient. The most advantage of LOMSEC is that by changing values of $r$, LOMSEC can create a series of various approximate solutions and as $r=\infty$ LOMSEC gives the same solution as the classical mean square error criterion does. Thus, LOMSEC can enable to obtain different accurate solutions whereas this is impossible for the classical criterion [31,32]. The main disadvantage of LOMSEC, however, is that the local domain of integration determined by the value of $r$ is unknown. To solve this problem the globallocal dual technique suggests that instead of finding a special value of $r$ one considers its 
varying in the global domain of integration. Thus, the constant replacement coefficient $k$ can be suggested as global mean value of all local replacement coefficients as follows

$$
k=\langle k(r)\rangle=\lim _{s \rightarrow \infty}\left(\frac{1}{s} \int_{0}^{s} k(r) \mathrm{d} r\right),
$$

where $\langle\cdot\rangle$ is used as the conventional notation for averaging operator of deterministic functions. Thus the use of the global-local dual technique for LOMSEC leads to a new criterion called global-local mean square error criterion (GLOMSEC) $[9,14]$.

\subsection{Weighted averaging dual technique}

In this subsection we consider again the problem of equivalent replacement and another dual technique is reviewed to show how the dual approach can be realized and new equivalent replacement coefficient can be obtained. Let we replace $A$ approximately with $k B$ where $k$ is found from the mean-square error criterion (1). Suppose that $A$ and $B$ are described by periodic functions $A(t)$ and $B(t)$ of $t$ belonging to the interval $[0,+\infty)$. Rewriting (1) in explicit form gives

$$
\lim _{T \rightarrow \infty} \frac{1}{T} \int_{0}^{T}(A(t)-k B(t))^{2} \mathrm{~d} t \rightarrow \min _{k} .
$$

Averaged values play major roles in the study of dynamic processes. The use of those values allows transforming varying processes to some constant characteristics that are much easier to be investigated. The conventional definition of averaged values, however, has some deficiencies, for example, if $(A(t)-k B(t))^{2}$ has terms whose integration in (23) are equal zero, the information contained in those terms is lost. For all terms that are harmonic functions $\cos (n t)$ and $\sin (n t)$ this observation is true. The dual approach to averaged values is an effective way to suggest an alternative choice for the conventional average value, namely the constant coefficient $1 / T$ in (23) can be extended to a weighting coefficient as function $h(t)$. Thus one gets so-called weighted mean square error criterion

$$
W(x(t))=\int_{0}^{\infty} h(t)(A(t)-k B(t))^{2} \mathrm{~d} t \rightarrow \min _{k},
$$

where the condition of normalization is satisfied

$$
\int_{0}^{\infty} h(t) \mathrm{d} t=1
$$

There isn't a general theory about weighting functions $h(t)$. The weighted dual technique is a possible way to construct weighting functions $h(t)$. First one introduces basic weighting coefficients as follows [15]:

A) Basic optimistic weighting coefficients

Basic optimistic weighting coefficients are increasing functions of $t$ and denoted as $O(t)$.

B) Basic pessimistic weighting coefficients

Basic pessimistic weighting coefficients are decreasing functions of $t$ and denoted as $P(t)$. 
C) Neutral weighting coefficients Basic neutral weighting coefficients, denoted as $N(t)$, are constants.

A dual weighting coefficient $h(t)$ is obtained as summation and/or product of basic weighting coefficients. Example is

$$
h(t)=\sum_{i=1}^{n}\left(\alpha_{i} O_{i}(t)+\beta_{i} P_{i}(t)+\gamma_{i} O_{i}(t) P_{i}(t)\right)+N(t),
$$

where $\alpha_{i}, \beta_{i}, \gamma_{i}$ are constants. The dual characteristics considered in (26) are the increasing and decreasing properties of basic weighting coefficients. These dual properties allow adjusting in dual weighted average values which can keep information about all the data of a given $\omega$-periodic function. The following particular dual weighting function is considered [15]

$$
h(t)=s^{2} \omega^{2} t e^{-s \omega t}, \quad s>0 .
$$

It is seen that the weighting coefficient (27), obtained as a product of the optimistic weighting coefficient $t$ and the pessimistic weighting coefficient $e^{-s \omega t}$ (see Fig. 1). It is worth mentioning that the dual weight function (27) is one-parameter function and it contains the conventional arithmetic weight as a particular case. In fact the dual weight function (27) equaling zero at only $t=0$ and $t=\infty$, has one maximal value at $t_{*}=1 /(s \omega)$, and then decreases to zero as $t \rightarrow \infty$. The dual weighting function (27) has maximal value at infinity when $s \rightarrow 0$. It means in this case the dual weighting function (27) approaches to the conventional arithmetic weight.

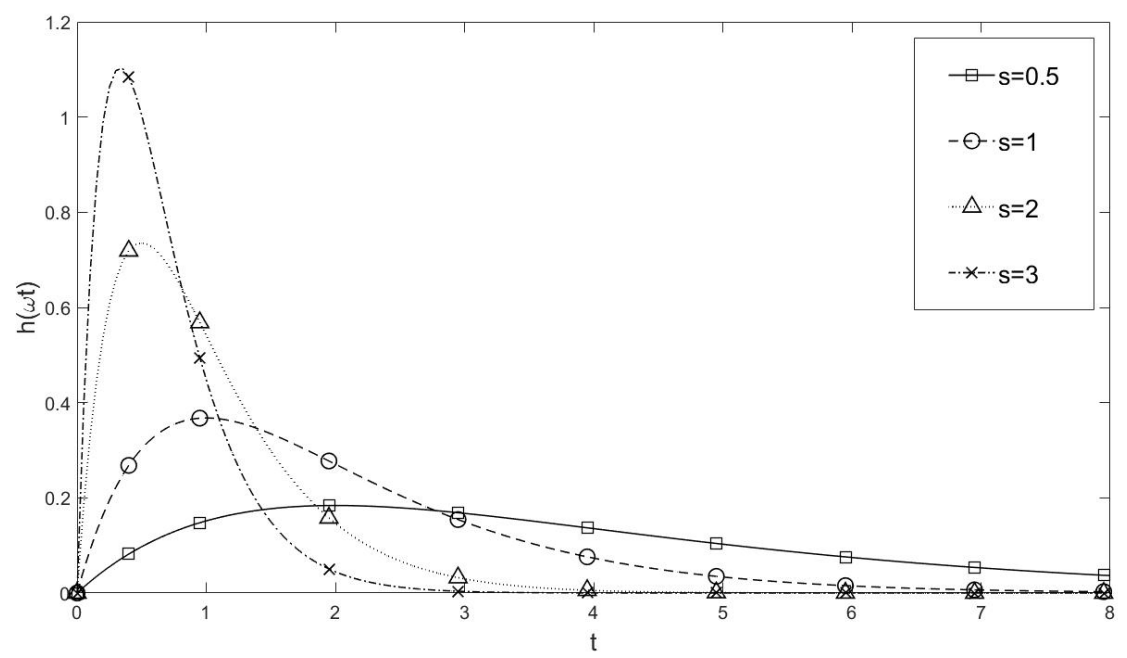

Fig. 1. Plot of dual weight function

Based on the weighting coefficient (27) a new weighted average value is proposed for a $\omega$-periodic function $f(\omega t)$

$$
W(f(\omega t))=\int_{0}^{\infty} s^{2} \omega^{2} t e^{-s \omega t} f(\omega t) \mathrm{d} t=\int_{0}^{\infty} s^{2} \phi e^{-s \phi} f(\phi) \mathrm{d} \phi .
$$


Using Laplace transformation Table one gets

$$
\begin{aligned}
& W(\cos n \omega t)=\int_{0}^{\infty} s^{2} \omega^{2} t e^{-s \omega t} \cos (n \omega t) \mathrm{d} t=\int_{0}^{\infty} s^{2} \phi e^{-s \phi} \cos (n \phi) \mathrm{d} \phi=s^{2} \frac{s^{2}-n^{2}}{\left(s^{2}+n^{2}\right)^{2}}, \\
& W(\sin n \omega t)=\int_{0}^{\infty} s^{2} \omega^{2} t e^{-s \omega t} \sin (n \omega t) \mathrm{d} t=\int_{0}^{\infty} s^{2} \phi e^{-s \phi} \sin (n \phi) \mathrm{d} \phi=s^{2} \frac{2 s n}{\left(s^{2}+n^{2}\right)^{2}} .
\end{aligned}
$$

As $\omega$-periodic functions $f(\omega t)$ can be expended into Fourier series and the dual weighted averaging operator (28) is linear one hence one can easy calculate (28) for any $\omega$-periodic functions $f(\omega t)$ by using (29) and (30). Indeed let $f(\omega t)$ be expended into a Fourier series

$$
f(\omega t)=f_{0}+\sum_{i=1}\left(f_{i c} \cos i \omega t+f_{i s} \sin i \omega t\right) .
$$

Substituting (31) into (27) using (9) and (10) one gets

$$
\begin{aligned}
W(f(\omega t)) & =W\left(f_{0}+\sum_{i=1}\left(f_{i c} \cos i \omega t+f_{i s} \sin i \omega t\right)\right) \\
& =f_{0}+\sum_{i=1}\left(f_{i c} W(\cos i \omega t)+f_{i s} W(\sin i \omega t)\right) \\
& =f_{0}+s^{2} \sum_{i=1}\left(f_{i c} \frac{s^{2}-i^{2}}{\left(s^{2}+i^{2}\right)^{2}}+f_{i s} \frac{2 i s}{\left(s^{2}+i^{2}\right)^{2}}\right) .
\end{aligned}
$$

In particular, putting $s=0$ in (32) leads to the conventional average value of $f(\omega t)$

$$
\langle f(\omega t)\rangle=\left.W(f(\omega t))\right|_{s=0}=f_{0} .
$$

The first advantage of the weighted dual average value (32) is clearly seen from (31)(33) that all the data $\left\{f_{i c}, f_{i s}\right\}$ of the function $f(\omega t)$ contained in (31) are preserved in the weighted dual average value (32) for $s \neq 0$ and will be lost in the conventional average value (33) for $s=0$. The second advantage is that one can regulate the value of $s$ to get the desired results from (32) depending on the considered problem.

\section{APPLICATIONS OF DUAL TECHNIQUES}

The equivalent linearization method of Krylov and Bogoliubov [33] that replaces a nonlinear system by an equivalent linear one was generalized to the case of nonlinear dynamic systems with random excitation by Kazakov and Caughey. In the theory of random vibration, the stochastic equivalent linearization method is a popular method since it preserves some essential properties of the original nonlinear system. The method has been described in numerous review articles [34,35], and was summarized in the monographs by Roberts and Spanos [29] and Socha [36]. The essential of the equivalent linearization method is how to find the linearization coefficients for a given nonlinear system. In the literature, several criteria of equivalent linearization have been suggested to 
define the linearization coefficients where the original version is the conventional criterion [29,34-36] that minimizes the mean-square of equation error. Despite the advantages, the main disadvantage of this criterion is that its accuracy decreases as the nonlinearity is increasing, in many cases it results in unacceptable errors. To improve the accuracy of the equivalent linearization method the dual mean-square error criterion is proposed in [2]. Application of the dual criterion to three nonlinear systems, namely Duffing, Van der Pol and Lutes-Sarkani oscillators, has shown an improved accuracy of the approximate solutions for cases where the nonlinearity is of intermediate level [4]. A possible reason may be the fact that the forward and return replacements would have different roles in adjusting the replacement error rather than being the same. The accuracy of the dual mean-square error criterion can be improved by introducing a weighted dual mean-square error criterion where the weight parameter $p$ is presented as a piecewise linear function of the squared correlation coefficient which is defined by the interpolation method of least squares from available exact solutions of several nonlinear restoring oscillators $[2,10,11]$. Introducing the weight parameter $p$ makes the weighted dual meansquare error criterion more flexible than the conventional and dual mean square error criteria. The empirical expression (15) for $p$ was given in [23]. The application to several nonlinear random systems has shown the improved accuracy of the proposed weighted dual equivalent linearization technique for a quite large range of nonlinearity. Extension of dual equivalent linearization technique to flutter analysis of two dimensional nonlinear airfoils was investigated in [16]. In $[20,21]$ the dual equivalent linearization is applied to the problem of thermal analysis of small satellites in Low Earth Orbit using one- and two-node models. To simplify the process of linearization, a preprocessing step in separating nonlinear terms of the original system is carried out to get an equivalent system in which each differential equation contains only one nonlinear term. Based on the dual mean square error criterion a closed form of linearization coefficients system is obtained and solved by a Newton-Raphson iteration procedure. It is shown that the solutions obtained from the dual criterion are in a good agreement with those obtained from the Grande's approach and Runge-Kutta algorithm.

The new development based on the dual approach to LOMSEC was suggested in [9, 14] where the authors proposed a global-local mean square error criterion (GLOMSEC) to single- and multi-degree-of-freedom stochastic nonlinear systems, considering local and global levels, and thus new values of linearization coefficients are obtained as global averaged values of all local linearization coefficients. The numerical results for several nonlinear systems under white noise excitation demonstrated a significant improvement in the accuracy of solutions, especially when the nonlinearity is strong.

The equivalent linearization method with the weighted averaging using weighting coefficient (27) was applied in [22] to analyze some vibrating systems with nonlinearities. The strongly nonlinear Duffing oscillator with third, fifth, and seventh powers of the amplitude, the other strongly nonlinear oscillators and the cubic Duffing with discontinuity are considered. The results obtained via this method are compared with the ones achieved by the Min-Max Approach (MMA), the Modified Lindstedt - Poincare Method (MLPM), the Parameter - Expansion Method (PEM), the Homotopy Perturbation Method (HPM) and 4th order Runge-Kutta method. 
Further developments of this weighted averaging have been presented in [24,25] for nonlinear systems appearing in practical engineering and physical problems. In [26,27] the equivalent linearization method with weighted averaging has been used to investigate the nonlinear vibration of microbeams based on the nonlinear elastic foundation through the modified couple stress theory [26] and the nonlinear vibration of nanobeams under electrostatic force through the nonlocal strain gradient theory [17]. The obtained results demonstrate that this method is very convenient for solving nonlinear equations.

Anh and Nguyen [6,7] suggested approximate analytical solutions of the optimal tuning ratio of the DVA for the $H_{\infty}$ optimization by using the idea of local averaging and dual replacement to the equivalent linearization method. Based on the idea of the weighted dual mean square error criterion, the authors of [8] give an analytical approach to the design of the three-element dynamic absorber for damped structures under ground motion by replacing the original damped structure by an equivalent undamped structure. Comparisons have been done to validate the accuracy of the obtained results. In [17] a simple method to determine the approximate analytical solutions of the nontraditional DVA when the damped primary structure is subjected to ground motion is proposed. The main idea is based on the dual mean square error criterion of the equivalent linearization method to replace approximately the original damped structure by an equivalent undamped one. Comparisons have been done to validate the effectiveness of the obtained results. The global-local approach is used in [18] to give approximate analytical solutions of $H_{\infty}$ optimization for all standard, three-element and non-traditional DVAs attached to damped primary structures. The study is based on the global-local criterion of the equivalent linearization method in order to replace approximately the original damped structure by an equivalent undamped one.

\section{SOME PERSPECTIVE ASPECS OF DUAL APPROACH}

In this section we suggest some further perspective research aspects of the dual approach. First the weighted dual mean square error criterion has been investigated for many stochastic nonlinear systems; however, it wasn't used to study deterministic nonlinear vibrational systems. Thus, numerical simulations can be carried out to check the accuracy of this criterion for free and forced vibrations in those systems. A new expression for the weighted parameter $p$ different from (16) may be proposed to improve the accuracy of the weighted dual mean square error criterion.

Similarly, the global-local mean square error criterion can be modified in order to be applicable to deterministic nonlinear vibrational systems. Second, comprehensive researches about the local averaging operator should be investigated for periodic functions. For $\omega$-periodic function $x(\omega t)$ the conventional averaged value of $x(\omega t)$ over one period is defined as

$$
\langle x(\omega t)\rangle=\frac{\omega}{2 \pi} \int_{0}^{2 \pi / \omega} x(\omega t) \mathrm{d} t=\frac{1}{2 \pi} \int_{0}^{2 \pi} x(\tau) \mathrm{d} \tau .
$$



value $r$

The local averaged value of $x(\omega t)$ is defined by replacing $2 \pi$ in (34) by a positive

$$
\langle x(\phi)\rangle_{r}=\frac{1}{r} \int_{0}^{r} x(\phi) \mathrm{d} \phi, \quad \phi=\omega t .
$$

If substituting the local averaging operator determined in (35) into the mean-square error criterion (1) for the equivalent replacement of $A(\omega t)$ by $k B(\omega t)$ one gets the local mean-square error criterion

$$
\left\langle e^{2}\right\rangle_{r}=\frac{1}{r} \int_{0}^{r}(A(\phi)-k B(\phi))^{2} \mathrm{~d} \phi \rightarrow \min _{k},
$$

which leads to

$$
k(r)=\frac{\int_{0}^{r} A(\phi) B(\phi) \mathrm{d} \phi}{\int_{0}^{r} B^{2}(\phi) \mathrm{d} \phi} .
$$

The behavior of the equivalent replacement coefficient $k(r)$ depending on the local value $r$ needs to be investigated. The question of how $r$ can be chosen is open and minimal values of $k(r)$ are ones of interest.

\section{CONCLUSIONS}

The study of nonlinear problems is of crucial importance not only in all areas of physics but also in engineering and in other disciplines. In particular, it appears that the analysis of vibration based on nonlinear mathematical models requires appropriate methods. Therefore, new methods for analysis of nonlinear oscillations always cause concern of scientists and technicians. Nature and life always contain dual inclinations. Those are perspectives that are contradictory, or complementary to each other. Therefore, scientific research also needs to reflect these properties. The main issue of a dual approach to a scientific problem is to always consider the two different (dual) aspects of the problem. This allows the study to become more harmonious and reflect the essence of the problem. In the dual approach a dual technique is one that can introduce two dual perspectives for the same problem in consideration.

This paper reviews key ideas of the reported researches on the dual approaches to the vibration analysis. Three types of dual techniques, namely, forward - return dual technique, global-local dual technique, weighted averaging dual technique for the problem of equivalent replacement are summarized. Different implements and realizations of dual techniques to nonlinear vibration analysis and design of dynamic absorbers are reviewed. Finally, the challenging issues based on the dual techniques are discussed. A number of possibilities for developing analytical techniques related to dual techniques 
are proposed. The review of the above studies shows that the dual approach is an appropriate one and the dual techniques are effective tools for studying random and deterministic nonlinear vibrational systems. Meanwhile the extension of the dual approach to other scientific problems is of high interest.

\section{ACKNOWLEDGEMENTS}

The paper is supported by Vietnam National Foundation for Science and Technology Development (NAFOSTED) under grant number 107.04-2018.12.

\section{REFERENCES}

[1] A. Fidlin. Nonlinear oscillations in mechanical engineering. Springer Science \& Business Media, (2005).

[2] N. D. Anh. Duality in the analysis of responses to nonlinear systems. Vietnam Journal of Mechanics, 32, (4), (2010), pp. 263-266. https://doi.org/10.15625/0866-7136/32/4/294.

[3] N. D. Anh. Dual approach to averaged values of functions. Vietnam Journal of Mechanics, 34, (3), (2012), pp. 211-214. https://doi.org/10.15625/0866-7136/34/3/2361.

[4] N. D. Anh, N. N. Hieu, and N. N. Linh. A dual criterion of equivalent linearization method for nonlinear systems subjected to random excitation. Acta Mechanica, 223, (3), (2012), pp. 645-654. https://doi.org/10.1007/s00707-011-0582-z.

[5] N. D. Anh, V. L. Zakovorotny, N. N. Hieu, and D. V. Diep. A dual criterion of stochastic linearization method for multi-degree-of-freedom systems subjected to random excitation. Acta Mechanica, 223, (12), (2012), pp. 2667-2684. https://doi.org/10.1007/s00707-012-0738-5.

[6] N. D. Anh and N. X. Nguyen. Extension of equivalent linearization method to design of TMD for linear damped systems. Structural Control and Health Monitoring, 19, (6), (2012), pp. 565573. https://doi.org/10.1002/stc.446.

[7] N. D. Anh and N. X. Nguyen. Design of TMD for damped linear structures using the dual criterion of equivalent linearization method. International Journal of Mechanical Sciences, 77, (2013), pp. 164-170. https://doi.org/10.1016/j.ijmecsci.2013.09.014.

[8] N. D. Anh, N. X. Nguyen, and L. T. Hoa. Design of three-element dynamic vibration absorber for damped linear structures. Journal of Sound and Vibration, 332, (19), (2013), pp. 4482-4495. https://doi.org/10.1016/j.jsv.2013.03.032.

[9] N. D. Anh, L. X. Hung, and L. D. Viet. Dual approach to local mean square error criterion for stochastic equivalent linearization. Acta Mechanica, 224, (2), (2013), pp. 241-253. https://doi.org/10.1007/s00707-012-0751-8.

[10] N. D. Anh, N. N. Linh, and N. Q. Hai. A weighted dual criterion for the problem of equivalent replacement. In Second International Conference on Vulnerability and Risk Analysis and Management (ICVRAM) and the Sixth International Symposium on Uncertainty, Modeling, and Analysis, Liverpool, UK, (2014), pp. 1913-1922, https://doi.org/10.1061/9780784413609.191.

[11] N. D. Anh and N. N. Linh. A weighted dual criterion for stochastic equivalent linearization method. Vietnam Journal of Mechanics, 36, (4), (2014), pp. 307-320. https://doi.org/10.15625/0866-7136/36/4/5106.

[12] N. D. Anh, I. Elishakoff, and N. N. Hieu. Extension of the regulated stochastic linearization to beam vibrations. Probabilistic Engineering Mechanics, 35, (2014), pp. 2-10. https://doi.org/10.1016/j.probengmech.2013.07.001. 
[13] N. D. Anh and N. X. Nguyen. Design of non-traditional dynamic vibration absorber for damped linear structures. Journal of Mechanical Engineering Science, 228, (1), (2014), pp. 45-55. https://doi.org/10.1177/0954406213481422.

[14] N. D. Anh, L. X. Hung, L. D. Viet, and N. C. Thang. Global-local mean square error criterion for equivalent linearization of nonlinear systems under random excitation. Acta Mechanica, 226, (9), (2015), pp. 3011-3029. https://doi.org/10.1007/s00707-015-1332-4.

[15] N. D. Anh. Dual approach to averaged values of functions: A form for weighting coefficient. Vietnam Journal of Mechanics, 37, (2), (2015), pp. 145-150. https://doi.org/10.15625/08667136/37/2/6206.

[16] N. M. Triet. Extension of dual equivalent linearization technique to flutter analysis of two dimensional nonlinear airfoils. Vietnam Journal of Mechanics, 37, (3), (2015), pp. 217-230. https://doi.org/10.15625/0866-7136/37/3/6474.

[17] N. D. Anh and N. X. Nguyen. Research on the design of non-traditional dynamic vibration absorber for damped structures under ground motion. Journal of Mechanical Science and Technology, 30, (2), (2016), pp. 593-602. https://doi.org/10.1007/s12206-016-0113-x.

[18] N. D. Anh, N. X. Nguyen, and N. H. Quan. Global-local approach to the design of dynamic vibration absorber for damped structures. Journal of Vibration and Control, 22, (14), (2016), pp. 3182-3201. https://doi.org/10.1177/1077546314561282.

[19] N. D. Anh, I. Elishakoff, and N. N. Hieu. Generalization of Seide's problem by the regulated stochastic linearization technique. Meccanica, 52, (4-5), (2017), pp. 1003-1016. https://doi.org/10.1007/s11012-016-0421-3.

[20] N. D. Anh, N. N. Hieu, P. N. Chung, and N. T. Anh. Thermal radiation analysis for small satellites with single-node model using techniques of equivalent linearization. Applied Thermal Engineering, 94, (2016), pp. 607-614. https://doi.org/10.1016/j.applthermaleng.2015.10.139.

[21] P. N. Chung, N. D. Anh, N. N. Hieu, and D. V. Manh. Extension of dual equivalent linearization to nonlinear analysis of thermal behavior of a two-node model for small satellites in Low Earth Orbit. International Journal of Mechanical Sciences, 133, (2017), pp. 513-523. https://doi.org/10.1016/j.ijmecsci.2017.09.011.

[22] N. D. Anh, N. Q. Hai, and D. V. Hieu. The equivalent linearization method with a weighted averaging for analyzing of nonlinear vibrating systems. Latin American Journal of Solids and Structures, 14, (9), (2017), pp. 1723-1740. https://doi.org/10.1590/1679-78253488.

[23] N. D. Anh and N. N. Linh. A weighted dual criterion of the equivalent linearization method for nonlinear systems subjected to random excitation. Acta Mechanica, 229, (3), (2018), pp. 1297-1310. https://doi.org/10.1007/s00707-017-2009-y.

[24] D. V. Hieu and N. Q. Hai. Analyzing of nonlinear generalized Duffing oscillators using the equivalent linearization method with a weighted averaging. Asian Research Journal of Mathematics, (2018), pp. 1-14. https://doi.org/10.9734/ARJOM/2018/40684.

[25] D. V. Hieu, N. Q. Hai, and D. T. Hung. The equivalent linearization method with a weighted averaging for solving undamped nonlinear oscillators. Journal of Applied Mathematics, 2018, (2018). https://doi.org/10.1155/2018/7487851.

[26] V.-H. Dang, D.-A. Nguyen, M.-Q. Le, and Q.-H. Ninh. Nonlinear vibration of microbeams based on the nonlinear elastic foundation using the equivalent linearization method with a weighted averaging. Archive of Applied Mechanics, (2019), pp. 1-20. https://doi.org/10.1007/s00419-019-01599-w.

[27] V.-H. Dang, D.-A. Nguyen, M.-Q. Le, and T.-H. Duong. Nonlinear vibration of nanobeams under electrostatic force based on the nonlocal strain gradient theory. International Journal 
of Mechanics and Materials in Design, (2019), pp. 1-20. https://doi.org/10.1007/s10999-01909468-8.

[28] J. Lee Rodgers and W. A. Nicewander. Thirteen ways to look at the correlation coefficient. The American Statistician, 42, (1), (1988), pp. 59-66. https://doi.org/10.1080/00031305.1988.10475524.

[29] J. B. Roberts and P. D. Spanos. Random vibration and statistical linearization. Dover Publications Inc., New York, (2003).

[30] N. D. Anh and M. Di Paola. Some extensions of Gaussian equivalent linearization. In International Conference on Nonlinear Stochastic Dynamics, (1995), pp. 5-15.

[31] L. X. Hung. Approximate analysis of some two-degree-of-freedom nonlinear random systems by an extension of Gaussian equivalent linearization. Vietnam Journal of Mechanics, 23, (2), (2001), pp. 95-109. https://doi.org/10.15625/0866-7136/9943.

[32] N. D. Anh and L. X. Hung. An improved criterion of Gaussian equivalent linearization for analysis of non-linear stochastic systems. Journal of Sound and Vibration, 1, (268), (2003), pp. 177-200. https://doi.org/10.1016/S0022-460X(03)00246-3.

[33] N. Krylov and N. Bogoliubov. Introduction to nonlinear mechanics. Princeton University Press, New York, (1943).

[34] S. H. Crandall. A half-century of stochastic equivalent linearization. Structural Control and Health Monitoring, 13, (1), (2006), pp. 27-40. https://doi.org/10.1002/stc.129.

[35] I. Elishakoff and S. H. Crandall. Sixty years of stochastic linearization technique. Meccanica, 52, (1-2), (2017), pp. 299-305. https://doi.org/10.1007/s11012-016-0399-x.

[36] L. Socha. Linearization methods for stochastic dynamic system. Lecture Notes in Physics, Springer, Berlin, (2008). 